\title{
SÍNTESE, CARACTERIZAÇÃO E APLICAÇÃO DE Ca-MOFs NA REMOÇÃO DO AZUL DE METILENO POR ADSORÇÃO
}

\author{
Olímpio J. Silva Junior ${ }^{a, *, \odot, ~ C a m i l l a . ~ V . ~ C . ~ M o r e i r a ~}{ }^{b}$, Arthur. F. F. Monteiro ${ }^{\mathrm{b}}$, Victor. E. R. Melo ${ }^{\mathrm{c}}$, João. B. L. Oliveira ${ }^{\mathrm{a}}$, \\ Joanna E. Kulesza ${ }^{\text {b }}$ Bráulio S. Barros ${ }^{\mathrm{d}}$ \\ a Instituto de Química, Universidade Federal do Rio Grande do Norte, 59064-741 Natal - RN, Brasil \\ ${ }^{\mathrm{b}}$ Departamento de Química Fundamental, Universidade Federal de Pernambuco, 50740-540 Recife - PE, Brasil \\ ${ }^{\mathrm{c}}$ Instituto Federal de Educação, Ciência e Tecnologia do Rio Grande do Norte, 59112-490 Natal - RN, Brasil \\ ${ }^{d}$ Departamento de Engenharia Mecânica, Universidade Federal de Pernambuco, 50740-550 Recife - PE, Brasil
}

Recebido em 19/08/2021; aceito em 19/11/2021; publicado em 17/01/2022

\begin{abstract}
SYNTHESIS, CHARACTERIZATION, AND APPLICATION OF Ca-MOFs IN THE REMOVAL OF METHYLENE BLUE VIA ADSORPTION. Metal-organic frameworks (MOFs) based on Calcium-terephthalate(Ca-MOFs) were synthesized by the sonochemical method and applied to remove methylene blue (MB) from an aqueous solution via adsorption. The samples were prepared and characterized by X-ray Powder Diffraction (PXRD), Attenuated Total Reflectance Fourier Transform Infrared Spectroscopy (ATR-FTIR), Thermal Analysis (TGA-DTA), and Scanning Electron Microscopy (SEM). The studied of the effect of the synthesis temperature on the crystallization of MOFs indicated two different crystal structures were identified, $\mathrm{Ca}(\mathrm{BDC})$ and $\mathrm{Ca}(\mathrm{BDC})(\mathrm{H} 2 \mathrm{O}) 3$. Observed that samples with a higher proportion of the $\mathrm{Ca}(\mathrm{BDC})$ phase showed greater dye adsorption capacity. The experimental data fit the pseudo-second-order kinetic model. The Langmuir and Freundlich adsorption models were applied to describe the equilibrium isotherms, and the isotherm constants were also determined. The adsorption equilibrium data preferably fit the Freundlich model. The samples were applied to methylene blue adsorption at pH 5.6, with maximum adsorption of 60.241 $\mu$ mol. $\mathrm{g}^{-1}$ and high reuse efficiency, reaching $97 \%$ of the initial adsorption capacity in the second reuse cycle.
\end{abstract}

Keywords: Ca-MOF; adsorption; methylene blue; coordination polymers.

\section{INTRODUÇÃO}

O intenso uso de corantes por parte de diversos setores industriais tem levado a um aumento significativo da contaminação dos efluentes gerados nas indústrias têxtil, de papel, de couro e plástico, farmacêutica, cosmética, dentre outras. ${ }^{1-5} \mathrm{Na}$ maioria dos casos o seu uso está relacionado ao tingimento ou mudança de cor a partir da utilização de soluções aquosas. O uso desse tipo de produto necessita de uma alta demanda de volume de água, e dessa forma, quantidades elevadas de águas residuais contaminadas são geradas e, geralmente, descartadas no meio ambiente sem o devido tratamento. Além de ser uma das principais causas de problemas agudos ocorridos no ecossistema, corantes que contêm a função azo podem apresentar ação tóxica, mutagênica e cancerígena. ${ }^{1,3,6}$

O azul de metileno (MB) é um desses corantes bastante utilizados pela indústria, um composto químico cíclico aromático de fórmula $\mathrm{C}_{16} \mathrm{H}_{18} \mathrm{~N}_{3} \mathrm{SCl}$ que em meio aquoso produz o cátion $\mathrm{C}_{16} \mathrm{H}_{18} \mathrm{~N}_{3} \mathrm{~S}^{+}$. A remoção natural dessa substância é difícil devido a sua estabilidade a luz, ao calor e aos agentes oxidantes., ${ }^{3,7}$ Vários métodos de remoção são utilizados em águas contaminadas, tais como métodos físicos, químicos e biológicos. No entanto, essas técnicas não são viáveis devido ao alto custo, além da formação de subprodutos de poluentes e requisição energética intensiva. ${ }^{1,3,6}$ As técnicas existentes para remoção de corantes de águas residuais incluem coagulação química/floculação, processos de oxidação, troca de íons e ultrafiltração. Um dos métodos que apresentam melhor resultado é o método físico-químico de adsorção, por ser uma técnica que dispensa altas temperaturas de operação, apresenta alta eficiência, viabilidade econômica, simples operação e possibilidade de utilização do material adsorvente em corantes diferentes. ${ }^{1-3}$

Uma grande variedade de materiais tem sido usada na adsorção do MB, tais como carvão ativado, ${ }^{3}$ zeólitas, ${ }^{7}$ sílica, ${ }^{9}$ redes metalorgânicas (MOFs), ${ }^{1-4,10-15}$ compósitos, ${ }^{16}$ casca de banana e bagaço de cana. ${ }^{6}$ O carvão ativado está entre os materiais que apresentam melhores resultados, ${ }^{3}$ pode ser sintetizado a partir de diversos métodos químicos e físicos, nos quais, dependendo da matéria-prima, método e condições de ativação, apresenta uma estrutura porosa definida. A adsorção usando carvão ativado é um método muito eficiente para a remoção de corantes orgânicos, o ponto fraco deste material é a sua difícil regeneração e o alto custo do processo, o que limita a sua aplicação ${ }^{1,6}$ e desperta o interesse de pesquisadores em novos materiais.

Polímeros de coordenação são uma nova classe de materiais, geralmente porosos, na qual átomos ou clusters metálicos são coordenados por ligantes orgânicos polifuncionais. Este tipo de material tem atraído o interesse de pesquisadores, ${ }^{17}$ se destacando como potencial alternativa para aplicação em muitas áreas da indústria, tais como o armazenamento de gases, ${ }^{18-24}$ processos de separação, ${ }^{25}$ sensores, ${ }^{26-29}$ catálise, ${ }^{30,31}$ eletrodos,${ }^{32}$ entre outros.

Neste trabalho, reporta-se a síntese de Ca-MOFs pelo método sonoquímico. No intuito de se consolidar a metodologia foi executado um planejamento fatorial para os fatores potência e tempo, com o objetivo de se determinar as condições ideais para a obtenção da estrutura desejada. Os materiais produzidos foram testados na adsorção do azul de metileno. Os testes realizados permitiram a determinação capacidade máxima de adsorção. Adicionalmente, foram investigados os mecanismos e a cinética de adsorção do processo.

*e-mail: olimpio.silva@ifrn.edu.br 


\section{PARTE EXPERIMENTAL}

\section{Materiais e métodos}

Todos os reagentes foram utilizados como recebidos, sem purificação adicional. Nitrato de Cálcio hexahidratado $\left(\mathrm{Ca}\left(\mathrm{NO}_{3}\right)_{2} \cdot 6 \mathrm{H}_{2} \mathrm{O}, 103 \%\right)$ de marca VETEC; ácido tereftálico (1,4- $\left.\mathrm{H}_{2} \mathrm{BDC}, 99 \%\right)$ de marca Sigma-Aldrich Chemistry, N,Ndimetilformamida (DMF, 99,8\%) de marca Dinâmica, Etanol $\left(\mathrm{C}_{2} \mathrm{H}_{5} \mathrm{OH}, 99,5 \%\right)$ de marca Atriom, trietilamina (TEA, $\mathrm{C}_{6} \mathrm{H}_{15} \mathrm{~N}, 99 \%$ ) de marca Cromoline Química Fina e azul de metileno trihidratado $\left(\mathrm{MB}, \mathrm{C}_{16} \mathrm{H}_{18} \mathrm{~N}_{3} \mathrm{SCl} .3 \mathrm{H}_{2} \mathrm{O}\right.$ ) da marca IMPEX.

As amostras foram analisadas por microscopia eletrônica de varredura (SEM) em um equipamento Hitachi, modelo TM3000 operado a $15 \mathrm{kV}$. As análises de difração de raios X de pó (PXRD) foram realizadas em um equipamento Bruker D2 Phaser trabalhando com radiação $\mathrm{Cu}-\mathrm{K} \alpha\left(\lambda_{\mathrm{Cu}}=1,542 \AA\right)$. As análises termogravimétricas (TGA) foram conduzidas em um sistema de análise térmica Shimadzu DTG-60H. As amostras foram aquecidas de 30 a $900{ }^{\circ} \mathrm{C}$ a uma taxa de $10{ }^{\circ} \mathrm{C} \mathrm{min}^{-1} \mathrm{sob}$ atmosfera de nitrogênio. As análises por Espectroscopia de Infravermelho por Transformada de Fourier com Refletância Total Atenuada (ATR-FTIR) foram realizadas em um espectrómetro Bruker Vertex 70/v. Para medição do pH utilizou-se um medidor da marca Hanna modelo pH21 e eletrodo HI1110 de corpo em vidro, junção única, enchimento a gel e uso geral. A síntese via método sonoquímico foi executada com o auxílio de um sonicador Sonics Vibra-Cell, modelo VC 505.

As concentrações de MB foram determinadas utilizando os valores de absorbância medidos a $665 \mathrm{~nm}$ por meio dos espectros de UV das soluções analisadas. Os valores de absorbância foram convertidos para valores de concentração de MB usando uma curva de calibração (Figura 1S). Cinco soluções padrão com pH 5,6 e 1, 2, 3, 4 e 5 ppm de MB foram preparadas. Em seguida, os espectros de UV destas soluções foram adquiridos, e os respectivos valores de absorbância utilizados para construir a curva de calibração. Todas os experimentos foram realizados em um espectrofotômetro Biospectro UV modelo SP-220.

\section{Síntese das MOFs de cálcio}

A síntese das Ca-MOFs foi realizada com base no procedimento reportado por Mazaj e colaboradores. ${ }^{33,34} \mathrm{Em}$ um procedimento típico, $0,26 \mathrm{~g}$ (1 mmol) de nitrato de cálcio hexahidratado foi dissolvido em $10 \mathrm{~mL}$ de água destilada (Solução A). A solução foi agitada magneticamente a uma temperatura de $318 \mathrm{~K}$ durante 5 minutos. Uma segunda solução foi preparada com $40 \mathrm{~mL}$ de DMF, 140,0 $\mu \mathrm{L}$ de TEA (base orgânica solúvel em DMF) e 0,18 g (1,08 mmol) de ácido tereftálico (Solução B). As soluções A e B foram misturadas sob agitação magnética por cerca de 10 minutos. Após esse período, a solução foi então submetida a um processamento ultrassônico. A aferição da temperatura da solução foi realizada com um termômetro digital. O produto sólido obtido foi centrifugado por cinco vezes usando uma rotação de $6000 \mathrm{rpm}$ durante cerca de 10 minutos, lavadas alternadamente com $15 \mathrm{~mL}$ de DMF e $15 \mathrm{~mL}$ de água destilada. Após a lavagem, as amostras foram secas em estufa a $358 \mathrm{~K}$ por quatro

Tabela 1. Parâmetros utilizados nas amostras CA1, CA2 e CA3

\begin{tabular}{cccc}
\hline Amostra & Potência $(\%)$ & Tempo (min) & Tempo total (min) \\
\hline CA1 & 40 & 30 & 50 \\
CA2 & 80 & 14 & 24 \\
CA3 & 60 & 30 & 50 \\
\hline
\end{tabular}

horas. Finalmente, as amostras foram maceradas com o auxílio de um almofariz e um pistilo de Agatha, armazenadas em tubos de Eppendorf e identificadas.

Os parâmetros sintéticos avaliados são apresentados na Tabela 1 . O procedimento sintético da amostra CA2 teve que ser interrompido aos 24 minutos devido à alta temperatura alcançada. A análise da variação da temperatura ao longo do tempo de síntese objetivou estabelecer uma possível correlação com a cristalização das estruturas metalorgânicas. Para o planejamento fatorial, ${ }^{35}$ realizou-se quatro experimentos $\left(2^{2}=4\right)$, mais o ponto central para as variáveis potência e tempo, Tabela 2. As amostras utilizadas foram preparadas com um "pulso ligado" de 6 s e um "pulso desligado" de 4 s e os valores dos parâmetros utilizados nos experimentos foram registrados na Tabela 3. Estudou-se os efeitos da centrifugação das amostras logo após a síntese e no dia seguinte, bem como a utilização do banho de gelo para limitar a temperatura da solução precursora durante o tratamento ultrassônico, Tabela 1S. Os códigos das amostras foram utilizados de acordo com os parâmetros sintéticos: a letra "B" foi utilizada para a síntese do banho de gelo, a letra " $X$ " para as sínteses que tiveram a centrifugação realizada no dia seguinte ( 24 h), a letra "P" seguida de um número indica a potência, a letra " $t$ " seguida de um número indica o tempo de síntese e "Ca" caracteriza a MOF de cálcio.

Tabela 2. Variáveis do planejamento fatorial

\begin{tabular}{lccc}
\hline Variáveis & - & 0 & + \\
\hline Potência (\%) & 40 & 60 & 80 \\
Tempo (min) & 15 & 30 & 45 \\
\hline
\end{tabular}

O efeito do $\mathrm{pH}$ na solução do MB por Ca-MOFs foi estudado para a faixa entre $2 \mathrm{e} 12$. O controle do $\mathrm{pH}$ foi feito por meio de soluções de $0,10 \mathrm{~mol} \mathrm{~L}^{-1}$ de $\mathrm{HCl}$ e $0,10 \mathrm{~mol} \mathrm{~L}^{-1}$ de $\mathrm{NaOH}$, usadas para acidificar ou alcalinizar, respectivamente, as soluções de corante. As curvas cinéticas foram obtidas usando as concentrações $66,8,133,7,267,4,401,1$ e 534,8 $\mu$ mol L ${ }^{-1}$, nas temperaturas de 293,303 e $313 \mathrm{~K}$, em solução de $\mathrm{pH}$ 5,6 de MB. Utilizou-se $50 \mathrm{~mL}$ da solução de MB, $50 \mathrm{mg}$ de Ca-MOF, durante cinco horas. $\mathrm{O}$ monitoramento da capacidade de adsorção foi obtido no tempo 0,5, 1, 2, 3 e $5 \mathrm{~h}$. As isotermas de Langmuir e Freundlich foram obtidas em ensaios com duração de três horas usando $10 \mathrm{~mL}$ de solução de MB (pH 5,6) e $10 \mathrm{mg}$ de Ca-MOF.

Tabela 3. Variações para cada experimento e a massa obtida.

\begin{tabular}{cccc}
\hline Amostra & Potência (\%) & Tempo (min) & Massa obtida (g) \\
\hline CA4 (1) & - & - & 0,013 \\
CA5 (2) & - & + & 0,008 \\
CA6 (3) & + & - & 0,127 \\
CA7 (4) & + & + & 0,044 \\
CA8 (5) & 0 & 0 & 0,051 \\
\hline
\end{tabular}

Os testes foram executados para concentrações de $66,8 \mu \mathrm{mol} \mathrm{L}^{-1}$, $133,7 \mu \mathrm{mol} \mathrm{L}{ }^{-1}, 267,4 \mu \mathrm{mol} \mathrm{L}-1,401,1 \mu \mathrm{mol} \mathrm{L}{ }^{-1}$ e $534,8 \mu \mathrm{mol} \mathrm{L}{ }^{-1}$, nas temperaturas de 293, 303 e $313 \mathrm{~K}$. Para o estudo de reuso do adsorvente foi utilizada uma solução de $133,9 \mu \mathrm{mol} \mathrm{L} \mathrm{L}^{-1}$ do corante (pH 5,6) em testes com duração de três horas a 293 K. A dessorção foi realizada lavando o material com $10 \mathrm{~mL}$ de etanol a $99,5 \%$, por três vezes. Na sequência a amostra foi seca em estufa a $333 \mathrm{~K}$. A capacidade de adsorção no equilíbrio Qe $\left(\mu \mathrm{mol} . \mathrm{g}^{-1}\right)$ foi calculada a partir da Equação 1:36-39

$$
\mathrm{Q}_{\mathrm{e}}=\frac{\left(\mathrm{C}_{\mathrm{o}}-\mathrm{C}_{\mathrm{e}}\right) \mathrm{V}}{\mathrm{m}}
$$


onde $\mathrm{C}_{0}$ e $\mathrm{C}_{\mathrm{e}}$ são as concentrações inicial e final de $\mathrm{MB}$, respectivamente, $\mathrm{V}(\mathrm{L})$ o volume da solução e $\mathrm{m}(\mathrm{g})$ a massa do adsorvente.

\section{RESULTADOS E DISCUSSÕES}

Pode-se observar um significativo aumento do rendimento obtido, cerca de $980 \%$ em massa de MOF, quando comparamos as amostras CA4 e CA6, Tabela 3. Este aumento foi alcançado mantendo-se o tempo de síntese no nível inferior e alterando a potência do nível inferior para o superior, caso da amostra CA6. Nas amostras CA5 e CA7, o tempo de síntese foi mantido no nível superior, a potência foi alterada do nível inferior para o superior, o que proporcionou um aumento de 550\% na massa obtida. Nas amostras CA4 e CA5, a potência de síntese foi mantida no nível inferior e alterou-se o tempo do nível inferior para o superior, neste caso foi observada a produção de apenas cerca de $62 \%$ para a massa obtida da MOF. Nas amostras CA6 e CA7, a potência usada foi mantida no nível superior e alterou-se o tempo do nível inferior para o superior, observandose uma produção de apenas cerca de $35 \%$ para a massa obtida da MOF. Verificou-se que as melhores condições para a produção das
MOFs estudadas implicam no uso do nível superior para a potência e inferior para o tempo.

Os difratogramas de raios-X das amostras CA1, CA2 e CA3 são apresentados na Figura $1 a$. As amostras de CA1 e CA2 apresentaram uma fase cristalina majoritária, $\mathrm{Ca}(\mathrm{BDC})$ (ficha CCDC 971026), ${ }^{33}$ e traços da fase $\mathrm{Ca}(\mathrm{BDC})\left(\mathrm{H}_{2} \mathrm{O}\right)_{3}$ (ficha CCDC 870928). ${ }^{40}$ Por outro lado, na amostra CA3 foi observada como fase principal $\mathrm{Ca}(\mathrm{BDC})$ $\left(\mathrm{H}_{2} \mathrm{O}\right)_{3}$. Uma comparação entre os difratogramas de todos as amostras produzidas indicou que as amostras sintetizadas em banho de gelo apresentaram majoritariamente a fase $\mathrm{Ca}(\mathrm{BDC})\left(\mathrm{H}_{2} \mathrm{O}\right)_{3}$, Figura $1 b$ e Figura $1 c$, enquanto nas amostras sintetizadas sem banho de gelo foram observadas duas fases: fase primária $\mathrm{Ca}(\mathrm{BDC})\left(\mathrm{H}_{2} \mathrm{O}\right)_{3}$ e fase secundária $\mathrm{Ca}(\mathrm{BDC})$, Figura $1 d$. Nas amostras sintetizadas sem banho de gelo a temperatura de síntese variou de 55 a $85^{\circ} \mathrm{C}$. As amostras preparadas com banho de gelo apresentaram temperatura abaixo de $40{ }^{\circ} \mathrm{C}$ e embora tenha ocorrido variação do tempo de síntese, a formação da fase $\mathrm{Ca}(\mathrm{BDC})\left(\mathrm{H}_{2} \mathrm{O}\right)_{3}$ ocorreu em todas as amostras.

A fase $\mathrm{Ca}(\mathrm{BDC})\left(\mathrm{H}_{2} \mathrm{O}\right)_{3}$ apresenta uma estrutura em cadeia "1D". Cada íon $\mathrm{Ca}^{+2}$ é coordenado por quatro átomos de oxigênio de grupos carboxilato de três ligantes BDC diferentes e quatro moléculas de água. Entre os dois grupos carboxilas do BDC há uma
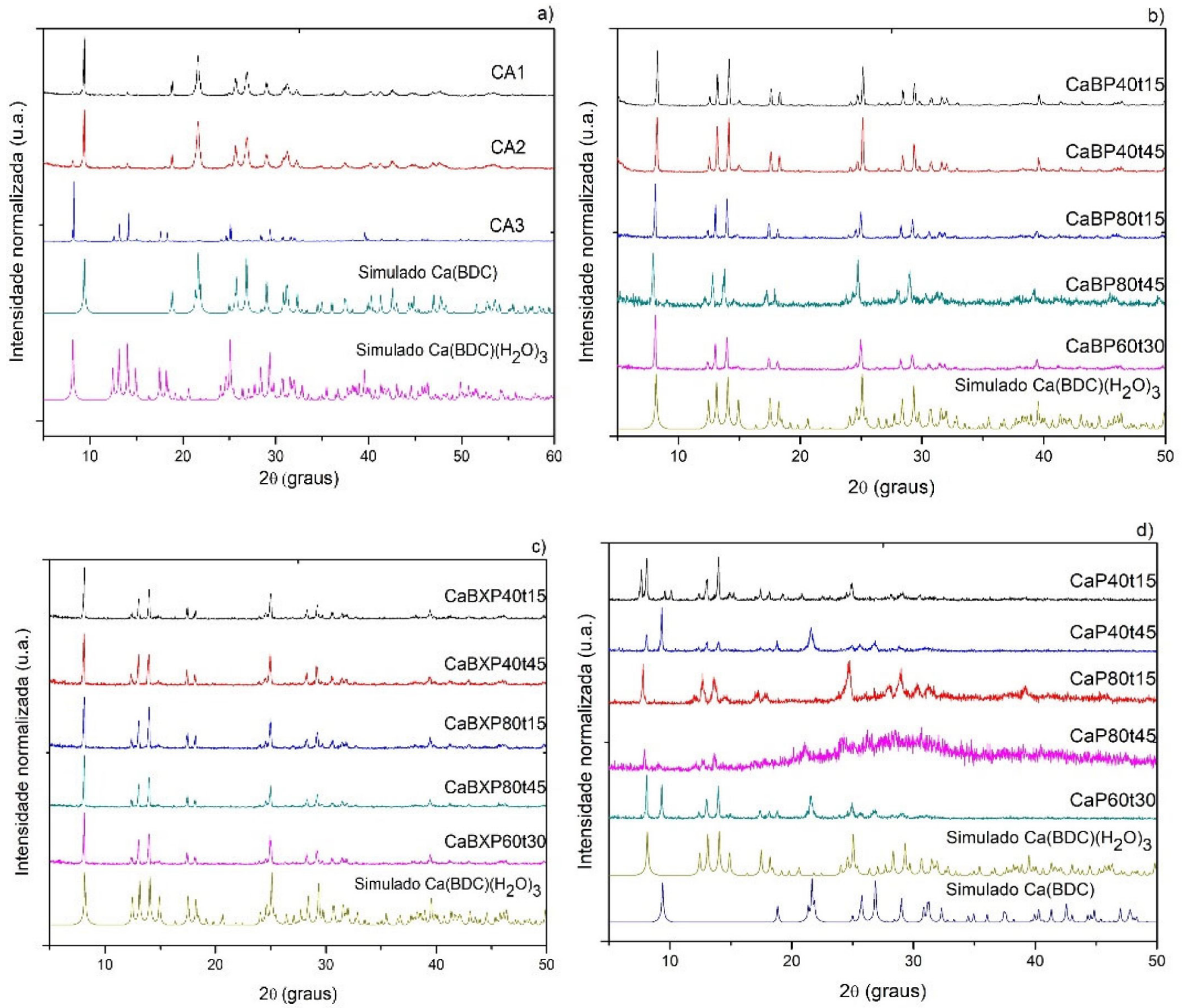

Figura 1. Difratogramas de raios-X das mostras CA1, CA2 e CA3 (a). Difratogramas de raios-X das amostras sintetizadas com banho de gelo e centrifugadas no mesmo dia (b), com banho de gelo e centrifugadas no dia seguinte (c) e sem banho de gelo e centrifugadas no mesmo dia 
ponte e um quelato envolvendo três íons $\mathrm{Ca}^{+2}$, o outro não participa da coordenação dos íons $\mathrm{Ca}^{+2}$. Cada poliedro de $\mathrm{CaO}_{8}$ compartilha duas faces com dois vizinhos e se estende para uma cadeia $\mathrm{Ca}-\mathrm{O}-\mathrm{Ca}$ inorgânica $1 \mathrm{D}$ ao longo do eixo cristalográfico $c$, Figura $2 a$. Existem grupos carboxilatos não coordenados em ambos os lados da cadeia. Devido à existência de grupos carboxilato expostos e moléculas de água coordenadas, interações significativas de ligações de hidrogênio entre as cadeias adjacentes finalmente resultaram em uma estrutura supramolecular $3 \mathrm{D} .{ }^{40} \mathrm{~A}$ estrutura $\mathrm{Ca}(\mathrm{BDC})$ apresenta cadeias formadas por octaédros $\mathrm{CaO}_{6}$ compartilhando duas arestas com seus vizinhos mais próximos e se estendendo ao longo do eixo $b$ e se ligam através de ligantes BDC ao longo do eixo $a$ originando uma estrutura cristalina 3-D não porosa, Figura $2 b$ A maioria das estruturas de Ca-MOFs possui centros de $\mathrm{Ca}^{+2}$ com números de coordenação mais altos que seis, onde o modo de coordenação de ponte quelante de grupos $\mathrm{COO}^{-}$ocorre preferencialmente quando $\mathrm{Ca}^{+2}$ está em ambiente com maior número de coordenação, normalmente de 7 a 9, enquanto o modo de ponte monodentada é encontrado na geometria octaédrica. $\mathrm{Na}$ estrutura do $\mathrm{Ca}(\mathrm{BDC})$ os grupos $\mathrm{COO}^{-}$tendem se a coordenar com $\mathrm{Ca}^{+2}$ no modo de ponte monodentada, o que causa a rotação do ligante de tereftalato por $\sim 90^{\circ}$ e a coordenação de maneira monodentada conectando duas cadeias $\mathrm{CaO}_{6}$ vizinhas. No entanto, devido ao seu raio iônico o íon $\mathrm{Ca}^{+2}$ exibe preferência por número de coordenação maior que seis e assim os íons, em ambiente octaédrico, apresentam uma alta tendência para ligação adicional de ligantes, no caso do $\mathrm{Ca}(\mathrm{BDC})$ seriam moléculas de água e sua transformação na fase $\mathrm{Ca}$ (BDC) $\left(\mathrm{H}_{2} \mathrm{O}\right)_{3}$. Embora a fase $\mathrm{Ca}(\mathrm{BDC})$ possua uma estrutura não porosa, apresenta flexibilidade estrutural nos processos de hidratação/ desidratação quando exposta a um ambiente úmido controlado transforma-se na fase hidratada $\mathrm{Ca}(\mathrm{BDC})\left(\mathrm{H}_{2} \mathrm{O}\right)_{3}$, e esta quando submetida ao vácuo, atmosfera inerte e/ou a temperatura elevada, transforma-se na estrutura $\mathrm{Ca}(\mathrm{BDC}){ }^{33}$

A transformação reversível $\mathrm{Ca}(\mathrm{BDC}) \leftrightarrow \mathrm{Ca}(\mathrm{BDC})\left(\mathrm{H}_{2} \mathrm{O}\right)_{3}$, Figura 3, é um processo que envolve a quebra e formação das ligações $\mathrm{Ca}-\mathrm{O}$, no qual ocorre a rotação do ligante $\mathrm{BDC}$, desconexão e protonação do grupo carboxilato de um lado, desconexão e formação parcial da ligação $\mathrm{Ca}-\mathrm{O}$ do outro lado do ligante BDC. Assim, a estrutura $\mathrm{Ca}(\mathrm{BDC})$ contendo o tereftalato conectado a quatro cadeias de $\mathrm{CaO}_{6}$ de maneira monodentada torna-se conectado a uma cadeia de $\mathrm{CaO}_{8}$ de maneira bidentada com o grupo $\mathrm{COO}^{-}$desprotonado de um lado, enquanto o carboxilato do outro lado fica protonado. Como já foi visto, esta estrutura supramolecular 3D é estabilizada por ligações de hidrogênio entre água coordenada e grupos carboxilato protonados. A desidratação desencadeia o mecanismo de transformação estrutural reverso: rompimento das ligações $\mathrm{Ca}-\mathrm{O}$ de um grupo carboxilato, rotação do ligante $\mathrm{BDC}$, desprotonação do grupo carboxilato e formação da ligação Ca-O..$^{33}$

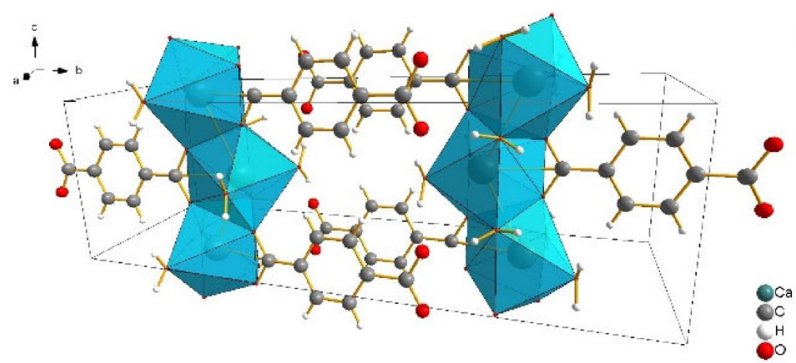

a)

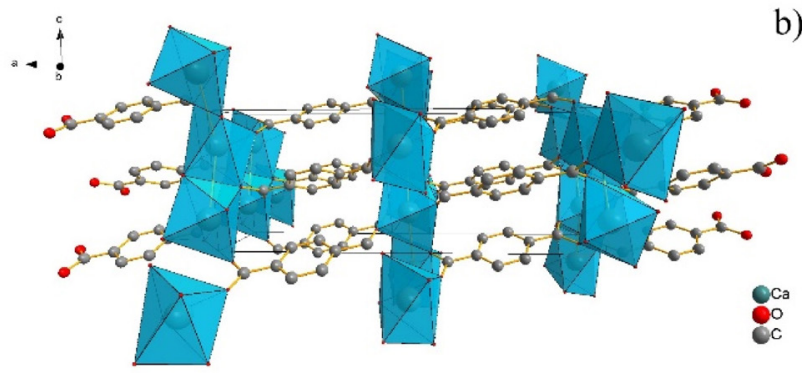

Figura 2. A célula unitária da estrutura $\mathrm{Ca}(\mathrm{BDC})\left(\mathrm{H}_{2} \mathrm{O}\right)_{3}$ consiste 104 átomos, monoclínico, $P 12_{1} / c 1, a=7.0982 \AA, b=21.6400 \AA, c=6.5856 \AA, \beta=92.2170^{\circ}$, no qual cada poliedro de $\mathrm{CaO}_{8}$ compartilha duas faces com dois vizinhos $e$ se estende para uma cadeia $\mathrm{Ca}-\mathrm{O}$-Ca inorgânica $1 \mathrm{D}$ ao longo do eixo cristalográfico c (a). A célula unitária da estrutura $C a(B D C)$ consiste 52 átomos, monoclínico, $C 12 / c 1, a=18.8433 \AA, b=5.3324 \AA, c=6.9596 \AA, \beta=87.0060^{\circ}$, com suas cadeias formadas por octaedros $\mathrm{CaO}_{6}$ compartilhando duas arestas com seus vizinhos mais próximos se estendendo ao longo do eixo b e se ligando ao BDC ao longo do eixo a originando uma estrutura cristalina $3-D$ não porosa $(b)$

As amostras de Ca-MOF sintetizadas com banho de gelo exibem claramente estrutura $\mathrm{Ca}(\mathrm{BDC})\left(\mathrm{H}_{2} \mathrm{O}\right)_{3}$, Figura $1 b$ e $c$, enquanto as amostras sintetizadas sem a utilização de banho de gelo exibem duas fases, Figura $1 d$. Devido a estrutura $\mathrm{Ca}(\mathrm{BDC})$ exibir estabilidade apenas em temperaturas elevadas, em atmosfera inerte ou no vácuo, enquanto em ambiente úmido, o $\mathrm{Ca}^{+2}$ se coordenará com mais três moléculas de água e se transformará em $\mathrm{Ca}(\mathrm{BDC})\left(\mathrm{H}_{2} \mathrm{O}\right)_{3}$.

As análises de FTIR, Figura 4, das amostras sugerem que existe uma coordenação de cálcio pelos grupos carboxilatos dos ligantes que são indicadas nos fortes picos de 1389 e $1433 \mathrm{~cm}^{-1}$ no estiramento simétrico $v_{\mathrm{s}}\left(\mathrm{COO}^{-}\right)$e para os picos 1510 e $1555 \mathrm{~cm}^{-1}$ no estiramento assimétrico $\mathrm{v}_{\mathrm{as}}\left(\mathrm{COO}^{-}\right)$. Isto é confirmado pela ausência do modo vibracional $v_{\mathrm{C}=\mathrm{O}}(\mathrm{COOH})$, referente à faixa de comprimento de onda $1680-1800 \mathrm{~cm}^{-1}$, indicando somente a presença de grupos carboxílicos desprotonados. Na figura $4 d$ é possível observar que o número de bandas presentes na região espectral próximo a 1400 $\mathrm{cm}^{-1}$ das amostras CaP40t45, CaP80t45 e CaP60t30 sugerem mais de

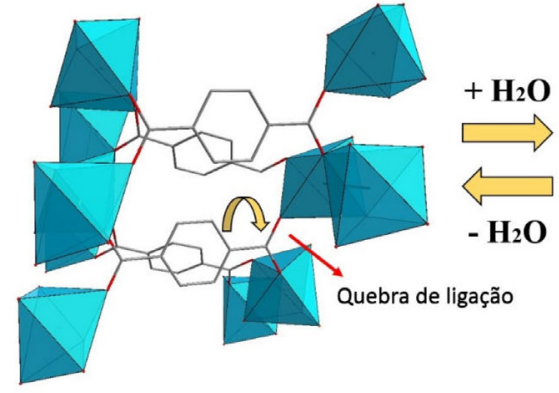

$\mathrm{Ca}(\mathrm{BDC})$

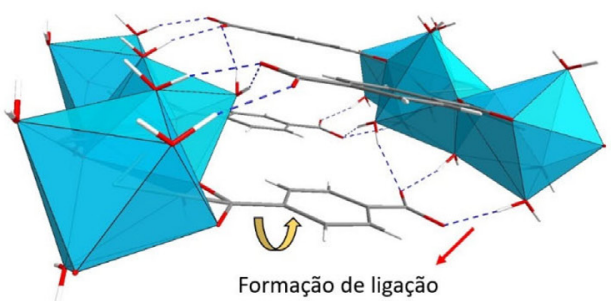

$\mathrm{Ca}(\mathrm{BDC})\left(\mathrm{H}_{2} \mathrm{O}\right)_{3}$

Figura 3. Esquema da transformação estrutural $\mathrm{Ca}(\mathrm{BDC}) \leftrightarrow \mathrm{Ca}(\mathrm{BDC})\left(\mathrm{H}_{2} \mathrm{O}\right)$ 
um modo de coordenação dos grupos carboxilatos e, de acordo com os difratogramas, Figura 1d, indicam o aparecimento da estrutura $\mathrm{Ca}(\mathrm{BDC})$. Nessas amostras é possível identificar as bandas $1330 \mathrm{e}$ $1350 \mathrm{~cm}^{-1}$ para os estiramentos simétricos $\mathrm{v}_{\mathrm{s}}(\mathrm{COO}-)$. A ligação com cátions metálicos interfere o espectro de infravermelho de compostos com grupos carboxilatos e a análise dessas alterações permite sugerir o tipo de coordenação ao íon metálico que pode ser identificado pela diferença no parâmetro $\Delta v\left(\mathrm{COO}^{-}\right)\left[\Delta=v_{\text {as }}\left(\mathrm{COO}^{-}\right)-v_{\mathrm{s}}\left(\mathrm{COO}^{-}\right)\right]$. Dessa maneira, o modo de coordenação entre os grupos carboxilatos e íons metálicos podem ser sugeridos de acordo com a regra empírica expressa como $\left[\Delta v\left(\mathrm{COO}^{-}\right)_{\text {[bidentado] }}\right]<\left[\Delta v\left(\mathrm{COO}^{-}\right)_{\text {[iônico] }}\right]<$ $\left[\Delta v\left(\mathrm{COO}^{-}\right)_{\text {[monodentado] }}\right] .{ }^{41} \mathrm{O} \Delta v=166 \mathrm{~cm}^{-1}$ resultante da diferença entre os picos 1555 e $1389 \mathrm{~cm}^{-1}$ indica a presença do modo de coordenação bidentado, de acordo com a descrição estrutural para a fase $\mathrm{Ca}(\mathrm{BDC})$ $\left(\mathrm{H}_{2} \mathrm{O}\right)_{3}$, e o aparecimento das bandas 1330 e $1350 \mathrm{~cm}^{-1}$ resulta no $\Delta v=225$ e $205 \mathrm{~cm}^{-1}$ que indica a presença do modo de coordenação monodentado para a fase $\mathrm{Ca}(\mathrm{BDC})$ nas amostras $\mathrm{CaP} 40 \mathrm{t} 45$, CaP80t45 e CaP60t30. ${ }^{42,43} \mathrm{O}$ pico mais largo na faixa de comprimento de onda entre $3250-3500 \mathrm{~cm}^{-1}$ está relacionada com a presença de moléculas de água, em que os picos mais largos próximos $3370 \mathrm{~cm}^{-1}$ estão relacionados a resíduos, enquanto que a faixa presente em $3294 \mathrm{~cm}^{-1}$ pode ser atribuída a moléculas de água coordenadas. ${ }^{33}$ É possível, dessa maneira, observar nos espectros a confirmação da presença de diferentes porções de água em todas as amostras sintetizadas, água superficial possivelmente causado pelo ambiente úmido, favorecendo a formação da fase $\mathrm{Ca}(\mathrm{BDC})\left(\mathrm{H}_{2} \mathrm{O}\right)_{3}$ em todas as amostras, e formação da fase $\mathrm{Ca}(\mathrm{BDC})$ apenas naquelas onde temperaturas mais elevadas foram alcançadas durante a síntese.

Nas imagens obtidas por MEV, Figura 2S, podemos observar uma morfologia muito semelhante àquela reportada por Mazaj. ${ }^{33,34} \mathrm{MAS}$ NMR, EXAFS, XANES As amostras sintetizadas com banho de gelo apresentaram partículas maiores em relação as partículas sintetizadas sem banho de gelo, indicando a influência que a temperatura tem sobre o crescimento das partículas.

A estabilidade térmica das Ca-MOFs foi estudada via análise termogravimétrica (TGA), Figura 5S. Nas respectivas curvas podemos observar uma primeira etapa de perda de massa que ocorre na faixa de temperatura entre 100 e $150{ }^{\circ} \mathrm{C}$, correspondente à liberação de moléculas de água coordenadas aos sítios metálicos. As amostras seguem estáveis até a temperatura próxima a $550{ }^{\circ} \mathrm{C}$, depois ocorrem uma segunda e uma terceira etapas de perda de massa correspondente ao início do colapso da estrutura devido decomposição do ligante orgânico, ácido tereftálico, levando a provável formação do óxido de cálcio.

As amostras sintetizadas foram submetidas a testes de adsorção para verificar a capacidade de adsorção de azul de metileno. Neste sentido, as MOFs sintetizadas sem banho de gelo apresentaram os maiores valores, Tabela $2 \mathrm{~S}$. Os resultados sugerem que a fase secundária $\mathrm{Ca}(\mathrm{BDC})$, apresentada nas amostras, pode ter sido responsável pelos melhores resultados. As MOFs de cálcio que
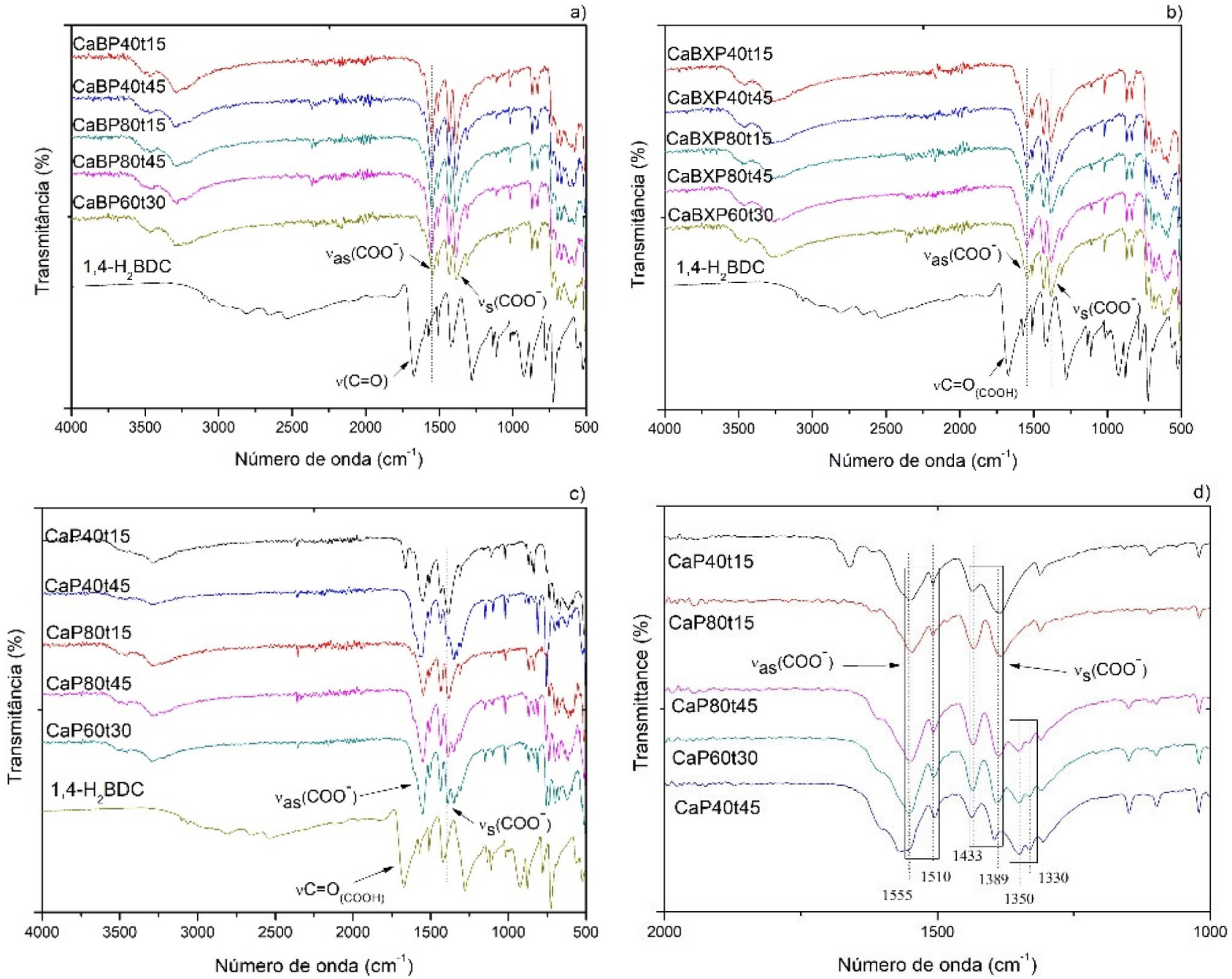

Figura 4. Espectro de FTIR das amostras sintetizadas com banho de gelo (a, b), sem banho de gelo (c) e bandas dos grupos carboxilatos das amostras sem banho de gelo $(d)$ 
apresentaram os melhores resultados foram as amostras CaP60t30 e CaP80t45. Assim, a amostra selecionada para utilização em todos os ensaios de adsorção foi a CaP60t30.

Os experimentos de adsorção foram realizados para uma solução de azul de metileno com concentração de $267,4 \mu$ mol.L $L^{-1}$ nas temperaturas de 293 K, 303 K, 313 K e 323 K, Figura 4S. O tempo máximo para cada ensaio foi de $5 \mathrm{~h}$. $\mathrm{O}$ aumento da temperatura provoca uma redução na capacidade de adsorção do MOF, por ser um processo exotérmico, no qual, para as condições de equilíbrio termodinâmico, o aumento da temperatura favoreceu a diminuição da adsorção. Assim, a temperatura mais adequada para realizar as etapas de investigação e com maior adsorção do corante pela MOF é a de $293 \mathrm{~K}$.

A capacidade de adsorção de azul de metileno da Ca-MOF aumenta rapidamente à medida que o $\mathrm{pH}$ aumenta de 2,21 para 3,23 , Figura 5S, e aumenta suavemente conforme o $\mathrm{pH}$ aumenta de 4,41 para 7,18. Observa-se um salto da capacidade de adsorção ao se atingir o pH 8,09 e valor máximo quando o pH alcança 11,58. Considerando que o MB é um corante catiônico, podemos concluir que a interação eletrostática desempenha um papel fundamental na adsorção, uma vez que sua carga superficial se torna negativa, pela adsorção de $\mathrm{OH}^{-}$presente no elevado meio básico, favorecendo a adsorção da espécie catiônica.

Em relação às isotermas de adsorção, dois modelos foram aplicados na análise dos dados para a identificação do modelo cinético em relação à natureza de adsorção do corante, os modelos

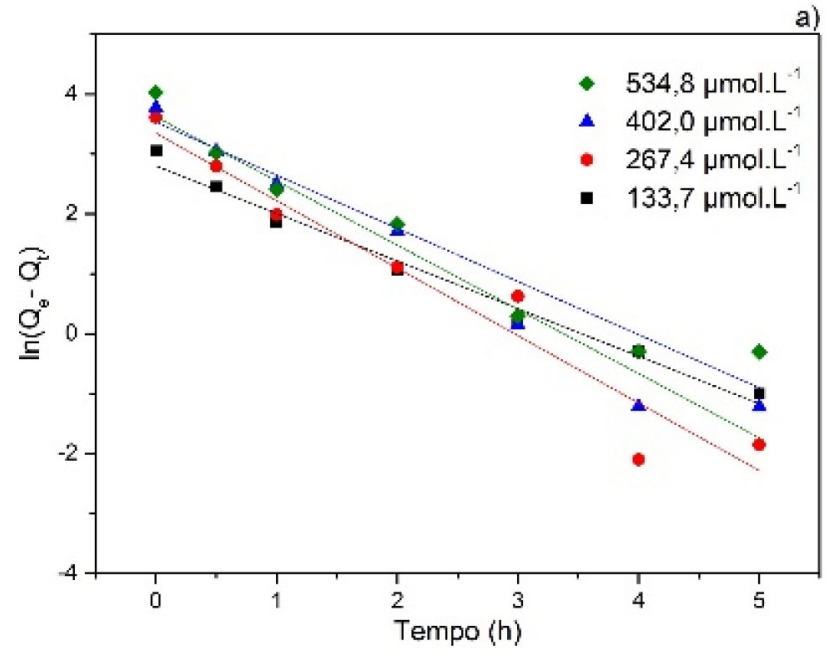

de pseudo-primeira e de pseudo-segunda ordem. ${ }^{1-3,14-16}$

$$
\begin{gathered}
Q_{t}=Q_{e}-Q_{e} e^{-k_{1} t} \\
\ln \left(Q_{e}-Q_{t}\right)=\ln Q_{e}-k_{1} t
\end{gathered}
$$

O modelo de pseudo-primeira ordem é representado pela equação não-linear, Equação 2, e também pode ser descrito por sua forma linearizada, Equação $3 .^{15,44-49} \mathrm{O} \mathrm{Q}_{\mathrm{e}}$ é a capacidade de adsorção em equilíbrio, $\mathrm{Q}_{\mathrm{t}}$ a capacidade de adsorção no tempo $\mathrm{t}, \mathrm{k}_{1}$ é a constante da taxa de adsorção, e t é o tempo de reação em um dado instante. $\mathrm{O}$ ajuste ao modelo mostra a dispersão entre os valores, Figura $5 a$. O modelo não é bem representativo para descrever o comportamento de adsorção do corante, Tabela 4.

Quando aplicado o modelo de pseudo-segunda ordem ${ }^{11,14,15,44-50}$ usou-se a equação linearizada, Equação 4, onde $Q_{\mathrm{e}}$ é a capacidade de adsorção em equilíbrio, capacidade de adsorção $Q_{t}$ no tempo $t, k_{2}$ a taxa de adsorção constante, e t é o tempo de reação em um dado instante. O modelo foi proposto por Ho e McKay,${ }^{51}$ onde é definido que a taxa de reação é dependente da quantidade de soluto adsorvido na superfície do adsorvente e da quantidade adsorvida no equilíbrio.

$$
\frac{t}{Q t}=\frac{1}{k_{2} Q_{e}^{2}}+\frac{t}{Q e}
$$

A Figura $5 b$ mostra o gráfico dos dados aplicados ao modelo pseudo-segunda ordem, no qual apresenta uma dispersão mínima,

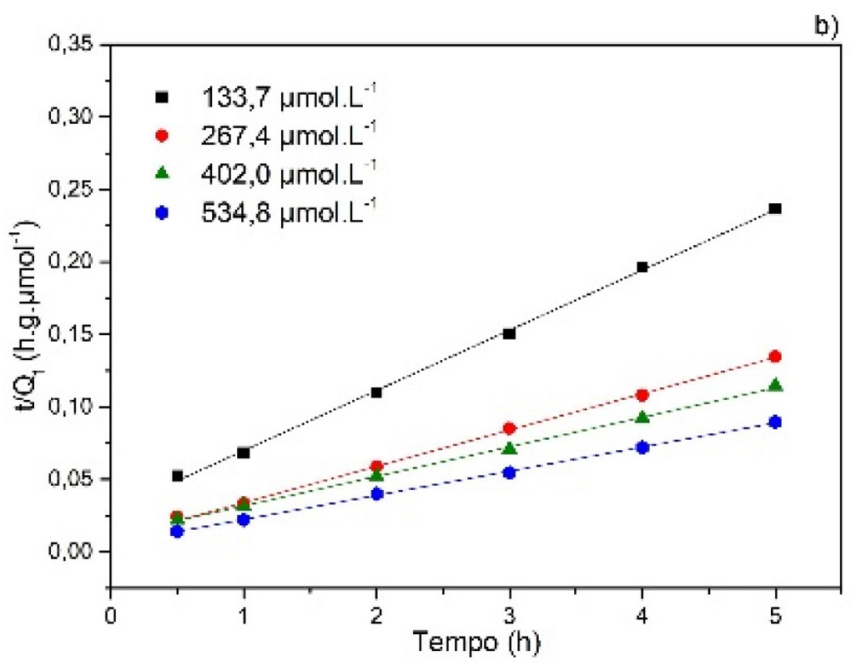

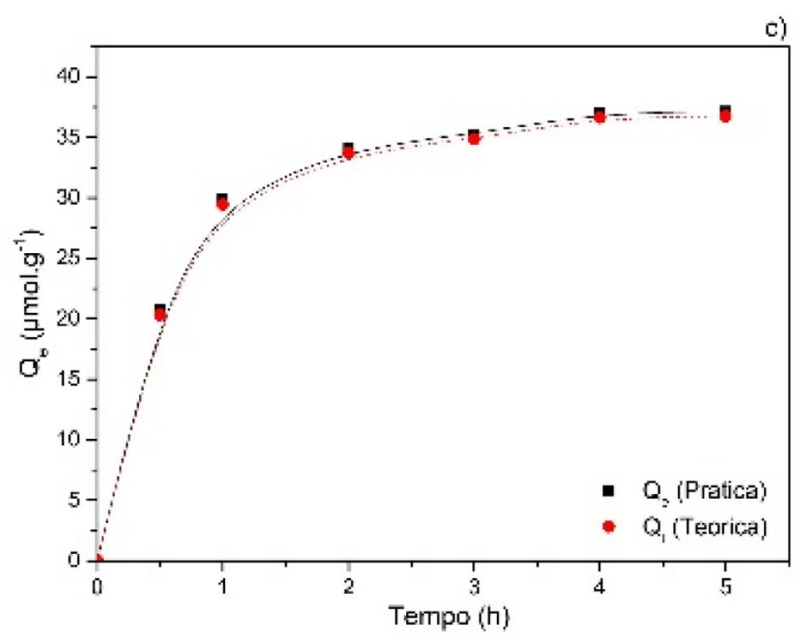

Figura 5. Linearização para o modelo de pseudo-primeira ordem na adsorção de MB a 293 K (a). Linearização para o modelo de pseudo-segunda ordem na adsorção de MB a $293 \mathrm{~K}$ (b). Curvas experimental e teórica para adsorção do azul de metileno a $293 \mathrm{~K}$ e 267,4 mol $\mathrm{L}^{-1}$ (c) 
Tabela 4. Valores para os modelos de pseudo-primeira e segunda ordem para a concentração de 267,4 mol L

\begin{tabular}{|c|c|c|c|c|c|}
\hline Modelo & & $293,0 \mathrm{~K}$ & $303,0 \mathrm{~K}$ & $313,0 \mathrm{~K}$ & $323,0 \mathrm{~K}$ \\
\hline \multirow[t]{3}{*}{ Primeira ordem } & $\mathrm{Q}_{\mathrm{e}}\left(\mu \mathrm{mol} \mathrm{g} \mathrm{g}^{-1}\right)$ & 30,072 & 24,301 & 15,319 & 18,078 \\
\hline & $\mathrm{K}_{1}$ & 1,1398 & 0,9721 & 0,9838 & 0,9200 \\
\hline & $\mathrm{R}^{2}$ & 0,9434 & 0,9285 & 0,9818 & 0,9596 \\
\hline \multirow[t]{5}{*}{ Segunda ordem } & $\mathrm{Q}_{\mathrm{e}}\left(\mu \mathrm{mol} \mathrm{g} \mathrm{g}^{-1}\right)$ & 40,323 & 32,051 & 21,930 & 19,646 \\
\hline & $\mathrm{K}_{2}\left(\mathrm{~L}^{-1} \mathrm{~h}^{-1} \mu \mathrm{mol}\right)$ & 0,062 & 0,075 & 0,113 & 0,023 \\
\hline & $\mathrm{R}^{2}$ & 0,9993 & 0,9991 & 0,9995 & 0,9939 \\
\hline & $\mathrm{h}\left(\mu \mathrm{mol} \mathrm{L} \mathrm{L}^{-1} \mathrm{~h}^{-1}\right)$ & 101,010 & 76,923 & 54,348 & 8,818 \\
\hline & $t_{1 / 2}(h)$ & 0,399 & 0,417 & 0,404 & 2,228 \\
\hline
\end{tabular}

Tabela 4. Para este modelo ainda são usadas as Equações 5 e 6 para obter o tempo de meia-vida, $\mathrm{t}_{1 / 2}$, e a taxa de adsorção inicial, $\mathrm{h}$, respectivamente. ${ }^{1}$

$$
\begin{aligned}
& t_{\frac{1}{2}}=k_{2} Q_{e} \\
& h=\frac{1}{k_{2} Q_{e}^{2}}
\end{aligned}
$$

Na Tabela 4, o coeficiente de determinação para o modelo pseudosegunda ordem é mais elevado e com maior precisão do que o modelo de pseudo-primeira ordem. Assim, o modelo de pseudo-segunda ordem foi confirmado como modelo cinético mais representativo para os ensaios realizados. Ao analisar e comparar os coeficientes de determinação para os dois modelos, Tabela 5, é possível estabelecer a melhor representatividade para o modelo cinético de adsorção do material. O valor calculado para a capacidade de adsorção foi maior, $40,323 \mu \mathrm{mol} \mathrm{g}{ }^{-1}$, para temperatura mais baixa, 293,0 K, contra a capacidade de adsorção observada à temperatura de 323,0 K, igual a $19,646 \mu \mathrm{mol} \mathrm{g} \mathrm{g}^{-1}$. A taxa de adsorção inicial também foi elevada para a temperatura mais baixa, enquanto a constante cinética está aumentando ao longo da temperatura. O tempo calculado no qual o sistema atingiu metade da sua capacidade de adsorção foi de cerca de 24 minutos à temperatura de 293,0 K. Os resultados mais significativos, para a concentração utilizada, são observados a uma temperatura de 293,0 K, nas isotermas de adsorção exibidas na Figura 5S.

Tabela 5. Coeficientes de determinação para os modelos de pseudo-primeira

\begin{tabular}{|c|c|c|c|c|}
\hline $\mathrm{C}_{0}\left(\mu \mathrm{mol} \mathrm{L}{ }^{-1}\right)$ & 133,7 & 267,4 & 401,1 & 534,8 \\
\hline Primeira ordem & 0,9792 & 0,9434 & 0,9712 & 0,9832 \\
\hline Segunda ordem & 0,9975 & 0,9993 & 0,9990 & 0,9994 \\
\hline
\end{tabular}
ordem e pseudo-segunda ordem em diferentes concentrações de MB a 293,0 K

Na Figura $5 c$ são apresentadas as curvas cinéticas experimental e teórica. A curva teórica foi plotada a partir do modelo de pseudosegunda ordem usando a Equação 7 em sua forma não-linearizada:

$$
Q_{t}=\frac{k_{2} Q_{e}^{2} t}{1+k_{2} Q_{e} t}
$$

onde $\mathrm{Q}_{\mathrm{e}}$ é a capacidade de adsorção de equilíbrio, ${ }^{1} \mathrm{Q}_{\mathrm{t}}$ e capacidade de adsorção no tempo $t, \mathrm{k}_{2}$ a constante de taxa de adsorção tempo de reação em um determinado instante. As curvas experimental e teórica apresentam uma alta compatibilidade, praticamente se sobrepondo.

As isotermas de adsorção são amplamente utilizadas na descrição do progresso da adsorção, além de serem utilizadas na investigação de mecanismos de adsorção e equilíbrio do processo. ${ }^{1}$ Os dados de equilíbrio para a Ca-MOF foram ajustados ao modelo de Langmuir e Freundlich. ${ }^{36,37,52} \mathrm{~A}$ aplicabilidade dos modelos isotérmicos ao comportamento de adsorção foi realizada a partir da avaliação do coeficiente de determinação. Esses modelos apresentam o estudo teórico descrevendo a interação sólido adsorvente/adsorvato na solução, apontando teoricamente, desta forma, a característica da superfície adsorvente e do mecanismo de adsorção. A Figura 6 $a$ apresenta os dados para capacidade de adsorção em função da concentração do corante no equilíbrio são plotados para análise da saturação da MOF e para obter a capacidade máxima de adsorção da rede metalorgânica para o corante. Com a diminuição da temperatura a capacidade de adsorção aumenta e revela que o processo de adsorção é exotérmico, além de que o aumento da concentração no qual é aplicado o adsorvente a capacidade adsortiva e a concentração no equilíbrio é maior.

A equação 8 descreve a forma não linearizada do modelo de Langmuir. ${ }^{1,11,53}$ A equação mais utilizada é sua forma linearizada, Equação 9, 2,3,14,15,44-49 aplicada à regressão linear e análise do coeficiente de determinação para avaliar a adequação ao modelo. Para equações lineares e não lineares $C_{e}$ é a concentração de equilíbrio, $Q_{e}$ é a capacidade de adsorção em equilíbrio, $\mathrm{Q}_{\mathrm{m}}$ é a capacidade máxima de adsorção, $\mathrm{K}_{\mathrm{L}}$ é a constante de Langmuir relacionada à energia de adsorção e afinidade dos sítios ligados. ${ }^{10}$

$$
\begin{gathered}
Q_{e}=\frac{K_{L} Q_{m} C_{e}}{1+K_{L} C_{e}} \\
\frac{C_{e}}{Q_{e}}=\frac{1}{Q_{m} K_{L}}+\frac{C_{e}}{Q_{m}}
\end{gathered}
$$

As isotermas de adsorção são exibidas utilizando o modelo de Langmuir, a partir de sua equação linear, com regressão linear a diferentes temperaturas. Os dados apresentados foram obtidos de diferentes concentrações e temperatura com o tempo de duração de cinco horas. Os dados do coeficiente de determinação para regressão linear são exibidos, além da capacidade máxima de adsorção e da constante de Langmuir, Tabela 6.

Quando o gráfico plotado, Figura 6b, da razão de concentração de equilíbrio e capacidade de adsorção $\left(\mathrm{C}_{\mathrm{e}} \cdot \mathrm{Q}_{\mathrm{e}}{ }^{-1}\right)$ versus concentração de equilíbrio $\left(\mathrm{C}_{\mathrm{e}}\right)$, os pontos não se ajustam ao modelo mostrando dispersão entre os valores de linearização. Assim, o modelo não é bem representativo para descrever a interação do corante MOF. A dispersão observada no gráfico é confirmada pelos valores do coeficiente de determinação calculados.

Outro modelo utilizado para análise da investigação dos mecanismos de adsorção e equilíbrio do processo é o de Freundlich..$^{37,39}$ Este modelo é amplamente utilizado em sistemas líquido-sólido onde, do ponto de vista energético, a interação entre a superfície do adsorvente e as espécies adsorvidas nos poros é heterogênea. $\mathrm{O}$ 
a)

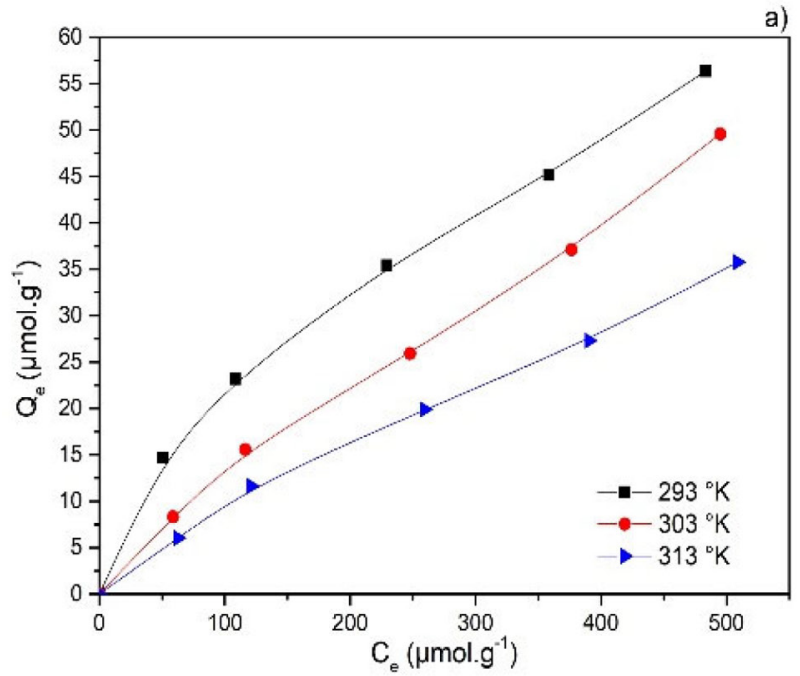

c)

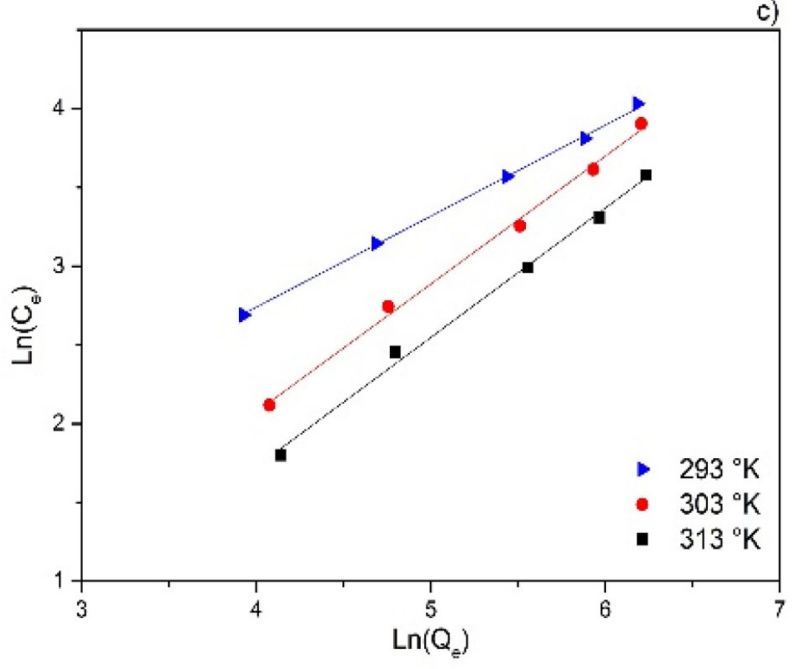

b)

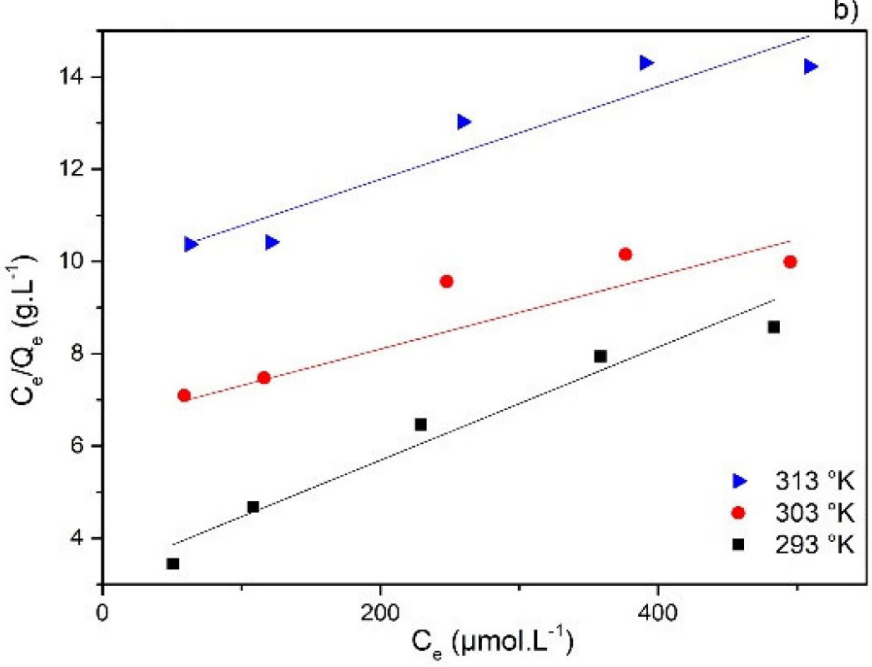

d)

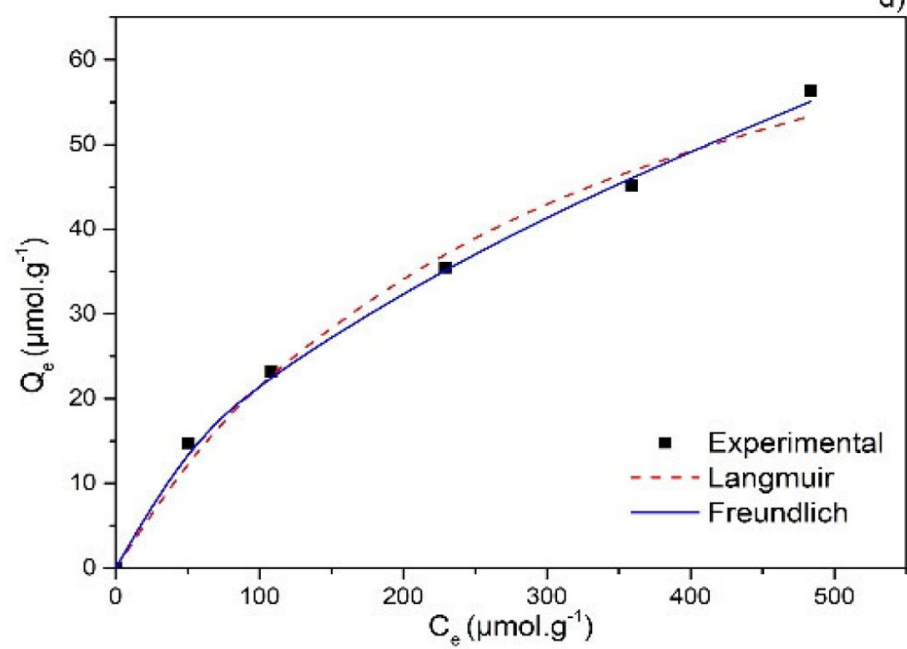

Figura 6. Isotermas de adsorção para diferentes temperaturas (a). Linearização de isotermas de adsorção pelo modelo de Langmuir (b). Linearização de isotermas de adsorção pelo modelo de Freundlich (c). Curva de adsorção calculada a partir do modelo isotérmico de Freundlich e Langmuir comparado com os valores experimentais para a mesma temperatura, $293 \mathrm{~K}(\mathrm{~d})$

Tabela 6. Valores calculados a partir do modelo de Langmuir e Freundlich a diferentes temperaturas

\begin{tabular}{llccc}
\hline Modelo & & $293,0 \mathrm{~K}$ & $303,0 \mathrm{~K}$ & $313,0 \mathrm{~K}$ \\
\hline Langmuir & $\mathrm{Q}_{\mathrm{m}}\left(\mu \mathrm{mol} \mathrm{g}^{-1}\right)$ & 83,333 & 140,845 & 98,039 \\
& $\mathrm{~K}_{\mathrm{L}}\left(\mathrm{L} \mathrm{mol}^{-1}\right)$ & 3643,540 & 1000,169 & 1041,954 \\
& $\mathrm{R}_{\mathrm{L}}$ & 0,8039 & 0,9443 & 0,9937 \\
& $\mathrm{R}^{2}$ & 0,9527 & 0,7612 & 0,8904 \\
\hline Freundlich & $\mathrm{Q}_{\mathrm{m}}\left(\mu \mathrm{mol} \mathrm{g}^{-1}\right)$ & 54,717 & 47,487 & 34,944 \\
& $\mathrm{~K}_{\mathrm{F}}\left(\mu \mathrm{mol} \mathrm{g}^{-1}\right)$ & 1,503 & 0,297 & 0,212 \\
& $\mathrm{n}$ & 1,719 & 1,223 & 1,220 \\
& $\mathrm{R}^{2}$ & 0,9981 & 0,9928 & 0,9956 \\
\hline
\end{tabular}

modelo é descrito pela Equação $10 .{ }^{1}$ A equação mais utilizada é a sua forma linearizada, Equação 11, aplicada à regressão linear e análise do coeficiente de determinação para avaliar a adequação ao modelo. Para $\mathrm{Q}_{\mathrm{e}}$ é a capacidade de adsorção em equilíbrio, $\mathrm{K}_{\mathrm{F}}$ é a constante de Freundlich, Ce é a concentração de equilíbrio e $n$ é a constante que indica a intensidade de adsorção, a afinidade do adsorvente pelo adsorvato..$^{14,15,39,44-49}$

$$
Q_{e}=K_{F} C_{e}^{\frac{1}{n}}
$$

$$
\ln Q_{e}=\ln K_{F}+\frac{1}{n} \ln C_{e}
$$

As isotermas de adsorção foram obtidas utilizando o modelo de Freundlich, a partir de sua equação linear, com regressão linear em diferentes temperaturas, Figura $6 c$. Os dados apresentados foram obtidos de diferentes concentrações e temperatura com o tempo de duração de cada ensaio de cinco horas. Os valores calculados a partir da linearização são mostrados na Tabela 7 e mostram valores para o coeficiente de determinação bastante próximos de 1,0 e superiores aos valores calculados para o modelo de Langmuir. A constante de equilíbrio, $\mathrm{K}_{\mathrm{F}}$, apresenta altos valores para a temperatura mais baixa, 293 K, concordando com o modelo de Freundlich para maior adsorção nas temperaturas mais baixas, sugerindo um processo exotérmico. Em relação ao processo favorável, o valor de $n$, relativo à intensidade de adsorção, indica condições favoráveis quando $1 / n$ está entre 0,1 e $1,0,{ }^{1}$ ou quando $n$ está entre 1,0 e 10,0, e não favorável quando $n$ é menor que $1,0{ }^{37,39}$ Assim, os valores calculados e exibidos para $n$ estão dentro do intervalo e apontam para processos favoráveis. ${ }^{36} \mathrm{~A}$ Figura $6 d$ mostra a curva de adsorção experimental e as curvas teóricas para os modelos de adsorção propostas por Langmuir e Freundlich.

A Tabela 6 mostra os valores para a capacidade de adsorção experimental e teórica para cada modelo, em que os valores 
calculados $\left(\mathrm{Q}_{\mathrm{e}, \mathrm{cal}}\right)$ a partir da equação não linear quando comparados com o valor experimental $\left(Q_{e, e x p}\right)$, apresentam resultados para o modelo de Freundlich mais próximos do valor encontrado para cada concentração. $\mathrm{O}$ valor do coeficiente de determinação confirma que o modelo que melhor se ajusta aos ensaios com a MOF e o corante é o modelo de Freundlich. Nos gráficos traçados para a curva experimental e a curva teórica, os pontos experimentais são mais próximos na curva de Freundlich em relação a de Langmuir. Em modelos cinéticos, como isotermas de adsorção, os valores de capacidade de adsorção mais significativos são aqueles para condições de baixa temperatura, sugerindo ser um processo adsorvente exotérmico.

Os parâmetros termodinâmicos, entalpia $\Delta \mathrm{H}\left(\mathrm{kJ} \mathrm{mol}^{-1}\right)$, energia livre $\Delta \mathrm{G}\left(\mathrm{kJ} \mathrm{mol}^{-1}\right)$ e $\Delta \mathrm{S}\left(\mathrm{J} \mathrm{mol}^{-1} \mathrm{~K}^{-1}\right)$ podem ser calculados a partir das Equações 12 a $14 .{ }^{54-56}$ Onde $\mathrm{R}\left(8.314 \mathrm{~J} \mathrm{~mol}^{-1} \mathrm{~K}^{-1}\right)$ é a constante dos gases, $\mathrm{T}(\mathrm{K})$ é a temperatura, $n$ a constante de heterogeneidade encontrada no modelo de adsorção de Freundlich. Para obter entalpia de adsorção, foi plotado o gráfico usando a equação linearizada, Equação 12 , como visto na Figura $6 \mathrm{~S}$, de $\operatorname{lnC}_{\mathrm{e}}$ versus $1 / \mathrm{T}\left(\mathrm{K}^{-1}\right)$.

$$
\begin{gathered}
\ln C_{e}=\frac{\Delta H}{R T}+\ln K \\
\Delta G=-n R T \\
\Delta S=\frac{\Delta H-\Delta G}{T}
\end{gathered}
$$

Tabela 7. Capacidade de adsorção calculada a partir do modelo de isoterma de Freundlich e Langmuir em relação aos valores experimentais para a temperatura de $293 \mathrm{~K}$

\begin{tabular}{cccc}
\hline Isotermas & & Freundlich & Langmuir \\
\hline $\mathrm{R}^{2}$ & & 0,9981 & 0,9527 \\
\hline $\begin{array}{c}\text { Concentração } \\
\left(\mu \mathrm{mol} \mathrm{L}^{-1}\right)\end{array}$ & $\mathrm{Q}_{\mathrm{e}, \mathrm{exp}}\left(\mu \mathrm{mol} \mathrm{g}{ }^{-1}\right)$ & $\mathrm{Q}_{\mathrm{e}, \text { cal }}\left(\mu \mathrm{mol} \mathrm{g}^{-1}\right)$ & $\mathrm{Q}_{\mathrm{e}, \text { cal }}\left(\mu \mathrm{mol} \mathrm{g}^{-1}\right)$ \\
\hline 66,952 & 14,719 & 14,739 & 12,848 \\
133,905 & 23,173 & 22,939 & 23,376 \\
267,809 & 35,305 & 35,470 & 37,664 \\
401,714 & 45,445 & 46,035 & 46,960 \\
535,619 & 56,344 & 54,754 & 52,915 \\
\hline
\end{tabular}

Os dados calculados são apresentados na Tabela 8, os valores encontrados para as entalpias são negativos, indicando que a adsorção do azul de metileno pela Ca-MOF é um processo exotérmico, como já previsto ao observar as isotermas, onde a capacidade de adsorção foi maior para temperaturas mais baixas e resultou em uma maior quantidade de corante adsorvido. A energia livre calculada foi entre $-20 \mathrm{Kj} \mathrm{mol}^{-1}$ e zero, refletindo numa adsorção física. ${ }^{10}$

A reutilização do adsorvente foi realizada utilizando $10 \mathrm{mg}$ de amostra CaP60t30 em $10 \mathrm{~mL}$ de solução de azul de metileno a 133,7 $\mu$ mol.L; , temperatura 293,0 K e pH de 5,6, durante 3 horas. A capacidade de adsorção se manteve elevada até o segundo ciclo, atingindo cerca de $97 \%$ da capacidade de adsorção inicial. Assim, a Ca-MOF apresentou elevada eficiência na remoção do corante azul de metileno em sua reutilização. Na Figura 7 são apresentados os espectros de FTIR da Ca-MOF após a adsorção e dessorção do MB. As bandas de vibrações características da MOF são observadas em todos os espectros, confirmando a estabilidade da estrutura $\mathrm{Ca}(\mathrm{BDC})$ $\left(\mathrm{H}_{2} \mathrm{O}\right)_{3}$ após os processos de adsorção e dessorção em meio aquoso. As amostras CaP60t30 e CaP80t45 apresentam bandas 1330 e $1350 \mathrm{~cm}^{-1}$, referente $\mathrm{a} \mathrm{v}_{\mathrm{s}}\left(\mathrm{COO}^{-}\right)$e modo de coordenação monodentada, conforme visto na Figura $4 d$ para a estrutura $\mathrm{Ca}(\mathrm{BDC})$, mas logo desaparecem após a adsorção do $\mathrm{MB}$ e isso se mantém na amostra de CaP60t30 após dessorção do corante, Figura 7. Isso pode indicar mudança no modo de coordenação do carboxilatos da fase $\mathrm{Ca}(\mathrm{BDC})$ quando em meio aquoso, na solução de $\mathrm{MB}$, e sua transformação para a estrutura porosa $\mathrm{Ca}(\mathrm{BDC})\left(\mathrm{H}_{2} \mathrm{O}\right)_{3}$.

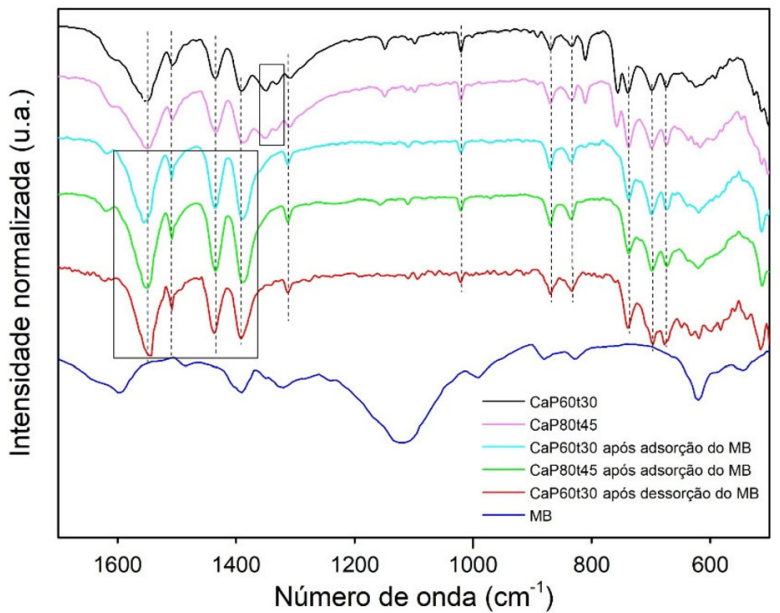

Figura 7. Espectros de infravermelho das amostras de Ca-MOF após adsorção e dessorção do azul de metileno

As bandas características do azul de metileno, 1591 e $1389 \mathrm{~cm}^{-1}$, podem ser observadas nos espectros da amostras após adsorção do azul de metileno a partir do aumento da intensidade dos picos exibidos na Figura 7. ${ }^{14,57} \mathrm{~A}$ adsorção pode ser explicada pela interação eletrostática entre corante e o adsorvente. ${ }^{5,11} \mathrm{O}$ corante azul de metileno apresenta carga positiva e dessa maneira experimentará uma interação eletrostática com adsorvente que apresente carga negativa, ânion de balanceamento de carga. ${ }^{3,11}$ Outra forma de interação está relacionada a presença de insaturações nas paredes dos canais da MOF, pertencentes ao ligante orgânico, havendo assim, dessa maneira, interação com os anéis aromáticos do azul de metileno, interações $\pi-\pi$, ocorrendo a adsorção. ${ }^{1,18,57}$

\section{CONCLUSÃO}

Ca-MOFs foram sintetizadas com sucesso pelo método

\begin{tabular}{|c|c|c|c|c|c|c|c|}
\hline \multirow{2}{*}{$\mathrm{C}_{0}\left(\mu \mathrm{mol} \mathrm{L}{ }^{-1}\right)$} & \multirow{2}{*}{$\frac{\Delta \mathrm{H}}{\left(\mathrm{kJ} \mathrm{mol}^{-1}\right)}$} & \multicolumn{3}{|c|}{$\Delta \mathrm{G}\left(\mathrm{kJ} \mathrm{mol}^{-1}\right)$} & \multicolumn{3}{|c|}{$\Delta \mathrm{S}\left(\mathrm{J} \mathrm{mol}^{-1} \mathrm{~K}^{-1}\right)$} \\
\hline & & $293 \mathrm{~K}$ & $303 \mathrm{~K}$ & $313 \mathrm{~K}$ & $293 \mathrm{~K}$ & $303 \mathrm{~K}$ & $313 \mathrm{~K}$ \\
\hline 66,952 & $-8,099$ & $-4,188$ & $-3,081$ & $-3,175$ & $-13,347$ & $-16,561$ & $-15,731$ \\
\hline 133,905 & $-4,255$ & $-4,188$ & $-3,081$ & $-3,175$ & $-14,524$ & $-14,045$ & $-13,596$ \\
\hline 267,809 & $-4,680$ & $-4,188$ & $-3,081$ & $-3,175$ & $-15,973$ & $-15,445$ & $-14,952$ \\
\hline 401,714 & $-3,246$ & $-4,188$ & $-3,081$ & $-3,175$ & $-11,080$ & $-10,714$ & $-10,372$ \\
\hline 535,619 & $-1,936$ & $-4,188$ & $-3,081$ & $-3,175$ & $-6,608$ & $-6,390$ & $-6,186$ \\
\hline
\end{tabular}
sonoquímico. Os principais parâmetros sintéticos foram estudados

Tabela 8. Parâmetros termodinâmicos de adsorção 
e um planejamento fatorial usado para estabelecer as condições mais favoráveis de otimização do processo de síntese. Os resultados sugerem que potências de ultrassom mais elevadas e menores tempos de reação são favoráveis a obtenção de estruturas metalorgânicas a base de Ca. Observou-se que uma maior potência de ultrassom tende a elevar a temperatura do meio reacional, levando a mudanças de fases. Os difratogramas de raios- $\mathrm{X}$ das amostras revelaram a formação de duas fases cristalinas distintas, $\mathrm{Ca}(\mathrm{BDC})$ e $\mathrm{Ca}(\mathrm{BDC})\left(\mathrm{H}_{2} \mathrm{O}\right)_{3}$, em função do tempo de síntese. As amostras que apresentaram maior proporção de fase $\mathrm{Ca}(\mathrm{BDC})$ também apresentaram maior capacidade de adsorção do corante. Os dados do estudo cinético de adsorção do azul de metileno se ajustaram bem ao modelo de pseudo-segunda ordem e a capacidade máxima de adsorção calculada considerando-se um $\mathrm{pH}$ de 5,6 foi de $60,241 \mu \mathrm{mol} \mathrm{g}^{-1}$. Para uma concentração de 534,8 $\mu$ mol. $\mathrm{g}^{-1}$ de azul de metileno a $293,0 \mathrm{~K}$, em um ensaio de cinco horas de duração, atingiu-se $93 \%$ da capacidade máxima de adsorção, ou seja, $56,073 \mu \mathrm{mol} \mathrm{g}{ }^{-1}$. Sobre a investigação do mecanismo de adsorção, o modelo de Freundlich foi o que melhor se aplicou, onde a interação entre a superfície do adsorvente e o corante nos poros ocorre de forma heterogênea. Em relação ao pH da solução, soluções com altos valores proporcionaram maior capacidade adsortiva. A reutilização do adsorvente mostrou-se eficiente até o seu segundo reciclo, com cerca de $97 \%$ de capacidade de adsorção em relação ao valor inicial.

\section{MATERIAL SUPLEMENTAR}

Algumas imagens dos sistemas utilizados neste trabalho estão disponíveis em http://quimicanova.sbq.org.br, na forma de arquivo PDF, com acesso livre.

\section{AGRADECIMENTOS}

Os autores agradecem às agências brasileiras CNPq e FACEPE (bolsa CNPq no 407445/2013-7 e bolsa PRONEX/FACEPE/CNPq no APQ-0675-1.06/14).

\section{REFERÊNCIAS}

1. Lin, S.; Song, Z.; Che, G.; Ren, A.; Li, P.; Liu, C.; Zhang, J.; Microporous Mesoporous Mater. 2014, 193, 27.

2. Liu, X.; Luo, J.; Zhu, Y.; Yang, Y.; Yang, S.; J. Alloys Compd. 2015, 648, 986.

3. Haque, E.; Jun, J. W.; Jhung, S. H.; J. Hazard. Mater. 2011, 185, 507.

4. Yang, Q.; Ren, S. S.; Zhao, Q.; Lu, R.; Hang, C.; Chen, Z.; Zheng, H.; Chem. Eng. J. 2018, 333, 49.

5. Haque, E.; Lee, J. E.; Jang, I. T.; Hwang, Y. K.; Chang, J. S.; Jegal, J.; Jhung, S. H.; J. Hazard. Mater. 2010, 181, 535.

6. Ma, J.; Huang, D.; Zou, J.; Li, L.; Kong, Y.; Komarneni, S.; J. Porous Mater. 2015, 22, 301.

7. Zhang, X.; Zhang, P.; Wu, Z.; Zhang, L.; Zeng, G.; Zhou, C.; Colloids Surfaces A Physicochem. Eng. Asp. 2013, 435, 85.

8. Raizada, A.; Ganguly, D.; Mankad, M. M.; J. Chem. Eng. Res. 2014, 2, 249.

9. Karim, A. H.; Jalil, A. A.; Triwahyono, S.; Sidik, S. M.; Kamarudin, N. H. N.; Jusoh, R.; Jusoh, N. W. C.; Hameed, B. H.; J. Colloid Interface Sci. 2012, 386, 307.

10. Yang, J. M.; J. Colloid Interface Sci. 2017, 505, 178.

11. Tong, M.; Liu, D.; Yang, Q.; Devautour-Vinot, S.; Maurin, G.; Zhong, C.; J. Mater. Chem. A 2013, 1, 8534.

12. Shen, T.; Luo, J.; Zhang, S.; Luo, X.; J. Environ. Chem. Eng. 2015, 3, 1372.

13. Haque, E.; Lo, V.; Minett, A. I.; Harris, A. T.; Church, T. L.; J. Mater. Chem. A 2014, 2, 193.
14. Tan, F.; Liu, M.; Li, K.; Wang, Y.; Wang, J.; Guo, X.; Zhang, G.; Song, C.; Chem. Eng. J. 2015, 281, 360.

15. Aslam, S.; Zeng, J.; Subhan, F.; Li, M.; Lyu, F.; Li, Y.; Yan, Z.; J. Colloid Interface Sci. 2017, 505, 186.

16. Tanhaei, M.; Mahjoub, A. R.; Safarifard, V.; Ultrason. Sonochem. 2018, $41,189$.

17. Furukawa, H.; Cordova, K. E.; O’Keeffe, M.; Yaghi, O. M.; Science (80). 2013, 341, 1230444.

18. He, Y.; Zhou, W.; Qian, G.; Chen, B.; Chem. Soc. Rev. 2014, 43, 5657.

19. Lotfi, R.; Saboohi, Y.; Comput. Theor. Chem. 2014, 1044, 36.

20. Dutta, S.; J. Ind. Eng. Chem. 2014, 20, 1148.

21. L.J.Murray, M.Dinca, J. R. L.; Chem. Soc. Rev. 2009, 38, 1294.

22. Li, J.; Cheng, S.; Zhao, Q.; Long, P.; Dong, J.; Int. J. Hydrogen Energy 2009, 34, 1377.

23. Bora, P. L.; Singh, A. K.; Int. J. Hydrogen Energy 2014, 39, 9293.

24. Langmi, H. W.; Ren, J.; North, B.; Mathe, M.; Bessarabov, D.; Electrochim. Acta 2013, 128, 368.

25. Li, J.; Sculley, J.; Zhou, H.; 2012, 869.

26. Li, Y.; Xie, M.; Zhang, X.; Liu, Q.; Lin, D.; Xu, C.; Xie, F.; Sun, X.; Sensors Actuators, B Chem. 2019, 278, 126.

27. Zhao, L.; Sun, K.; Youliwasi, N.; Guo, H.; Yang, G.; Jiao, F.; Dong, B.; Chai, Y.; Mintova, S.; Liu, C.; Appl. Surf. Sci. 2019, 470, 91.

28. Cao, Y.; Wang, L.; Shen, C.; Wang, C.; Hu, X.; Wang, G.; Sensors Actuators, B Chem. 2019, 283, 487.

29. Zhu, J.; Xia, T.; Cui, Y.; Yang, Y.; Qian, G.; J. Solid State Chem. 2019, $270,317$.

30. Bagherzadeh, M.; Ashouri, F.; Đaković, M.; J. Solid State Chem. 2015, $223,32$.

31. Silva Junior, O. J.; Monteiro, A. F. F.; Oliveira, J. B. L.; Araújo, A. M. U.; Silva, D. G.; Kulesza, J.; Barros, B. S.; Mater. Chem. Phys. 2019, 235, 121737.

32. Li, C.; Hu, X.; Hu, B.; Electrochim. Acta 2017, 253, 439.

33. Mazaj, M.; Mali, G.; Rangus, M.; Žunkovič, E.; Kaučič, V.; Zabukovec Logar, N.; J. Phys. Chem. C 2013, 117, 7552.

34. Mazaj, M.; Zabukovec Logar, N.; Cryst. Growth Des. 2015, 15, 617.

35. Cunico, M. W. M. W. M.; Miguel, O. G. G.; Zawadzi, S. F.; PeraltaZamora, P.; Volpato, N.; Visão Acadêmica 2008, 9, 23.

36. Raymond, A. W. W. A.; Water Quality and Treatment: a Handbook of Community; Letterman, R. D., Ed.; 5th ed.; McGRAW-HILL, INC.: New York, 1999.

37. Nascimento, R. F. do; Lima, A. C. A. de; Vidal, C. B.; Melo, D. de Q.; Raulino, G. S. C.; Adsorção: aspectos téoricos e aplicações ambientais; Imprensa Universitária, 2014.

38. Luo, X. P.; Fu, S. Y.; Du, Y. M.; Guo, J. Z.; Li, B.; Microporous Mesoporous Mater. 2017, 237, 268.

39. Libânio, M.; Fundamentos de Qualidade e Tratamento de Água by Editora Átomo; 4th ed.; Editora Átomo: Campinas, 2016.

40. Zhang, X.; Huang, Y. Y.; Zhang, M. J.; Zhang, J.; Yao, Y. G.; Cryst. Growth Des. 2012, 12, 3231.

41. Wang, M.; Li, H.; Huang, X.; Yi, L.; J. Vinyl Addit. Technol. 2014, $20,1$.

42. Phillips, R. J.; Deacon, G. B.; Coord. Chem. Rev. 1980, 33, 227.

43. Sutton, C. C. R.; Da Silva, G.; Franks, G. V.; Chem. - A Eur. J. 2015, 21 , 6801.

44. Vardikar, H. S.; Bhanvase, B. A.; Rathod, A. P.; Sonawane, S. H.; Mater. Chem. Phys. 2018, 217, 457.

45. Sharifpour, E.; Khafri, H. Z.; Ghaedi, M.; Asfaram, A.; Jannesar, R.; Ultrason. Sonochem. 2018, 40, 373.

46. Zhao, S.; Chen, D.; Wei, F.; Chen, N.; Liang, Z.; Luo, Y.; Ultrason. Sonochem. 2017, 39, 845.

47. Dastkhoon, M.; Ghaedi, M.; Asfaram, A.; Goudarzi, A.; Mohammadi, S. M.; Wang, S.; Ultrason. Sonochem. 2017, 37, 94.

48. Sharifpour, E.; Haddadi, H.; Ghaedi, M.; Ultrason. Sonochem. 2017, 36, 236. 
49. Tadjarodi, A.; Moazen Ferdowsi, S.; Zare-Dorabei, R.; Barzin, A. Ultrason. Sonochem. 2016, 33, 118

50. Xiao, X.; Zhang, F.; Feng, Z.; Deng, S.; Wang, Y.; Phys. E LowDimensional Syst. Nanostructures 2015, 65, 4.

51. Ho, Y. S.; McKay, G.; Process Biochem. 1999, 34, 451.

52. Moreno-castilla, C.; Carbon N. Y. 2004, 42, 83.

53. Langmuir, I.; J. Am. Chem. Soc. 1912, 34, 860.

54. Huang, J.; Huang, K.; Liu, S.; Luo, Q.; Shi, S.; J. Colloid Interface Sci. 2008, $317,434$.
55. Jianguo, C.; Aimin, L.; Hongyan, S.; Zhenghao, F.; J. Hazard. Mater. 2005, 124, 173.

56. Wang, H. ling; Fei, Z. hao; Chen, J. long; Zhang, Q. xing; Xu, Y. hua; J. Environ. Sci. 2007, 19, 1298

57. Fu, J.; Chen, Z.; Wang, M.; Liu, S.; Zhang, J.; Zhang, J.; Han, R.; Xu, Q.; Chem. Eng. J. 2015, 259, 53. 